\title{
The Reanalysis about College students' Autonomous Learning and Its Influence Factors
}

\author{
Meng Weijia \\ Military Education Theory \\ Aviation University of Air Force \\ Changchun, China \\ myg123789@sina.com
}

\author{
Lv Yang \\ Military Education Theory \\ Aviation University of Air Force \\ Changchun, China
}

\author{
Du Han \\ Military Education Theory \\ Aviation University of Air Force \\ Changchun, China
}

\begin{abstract}
Autonomous learning is a kind of learning mode widely advocated in today's education sector. On the basis of previous studies, directional research method is applied in this paper to analyze the influence factors of China's college students' autonomous learning. The following factors are innovatively designed and completed in this paper: peer comparison, showing opportunities and self-image. At the same time, a theory model assumption is put forward of college students' autonomous learning affecting factors, and according to the assumption strategies are provided to improve college students' autonomous learning ability.
\end{abstract} factors

Keywords—college students; autonomous learning; influence

\section{INTRODUCTION}

In the 1960s, the west pedagogy and psychology researchers began to put forward the autonomous learning theory, of which the core is to answer the question of how to make students become the master of their own learning. In the early 1970s, the United Nations Educational Scientific and Cultural Organization said in the report Learn to survive - the world education's today and tomorrow that, "Tomorrow's illiterate will not be illiterate people, but the people who don't know how to learn." Later, in 1996, the International Council on Education in the 21st Century in the report "EducationWealth Contain it", which was submitted to the UNESCO, made the idea of "learning to cognitive" one of the four pillars of social education. And it is clear that the purpose of education is to make students master the cognitive means cognitive method and learning method, rather than obtaining a systematic knowledge. The reason why the UNESCO stressed the importance of autonomous learning is that autonomous learning is vitally important to the development of the society, the education reform and individual.

In China the famous educator Tao Xingzhi has pointed out, "I think a good teacher is not to teach knowledge, not to teach students, but to teach students to learn." As to how to learn, it needs the teacher's guidance to make "teaching" be "don't need to teach". The fact that "Autonomous learning" was put forward in China reflects, on the one hand, the new achievements in the study of Chinese learning domain theory, and on the other hand, a series of new fundamental problems that were discovered in the current China's entire education reform. Higher education as the key part of training talents cannot be ignored. Training high-quality talents with innovative spirit and practical ability as the core is the purpose of China's higher education reform, and training modern college students adapting to the requirements of the learning society is the key content of "Teaching Contents and Curriculum System Reform Plan of Higher Education Facing the 21st Century". It is also the key to carry out quality education and college teaching reform.

\section{AutOnOmous LeARning THEORIES}

\section{A. The Operating Doctrine of Autonomous Learning}

Operating school represented by Skinner believes that autonomous learning is essentially a kind of operational behavior, which is based on external reward or punishment and makes a responsive reaction. Autonomous learning contains three sub-processes: self-monitoring, self-directed and selfreinforcing. Self-monitoring refers to the students' observation, review and evaluation for their own learning process; Selfdirected refers to the students take those behaviors that tend to study results, including making study plan, choosing the appropriate learning methods and organizing learning environment, etc.; Self-reinforcing refers to students according to the learning results get reward or punishment to facilitate the active learning process to maintain or promote.

\section{B. The Humanism View of Autonomous Learning}

Since the 1980s, some humanistic psychologists made an in-depth and systematic analysis to the inner psychological mechanism of autonomous learning. McCombs thought that autonomous learning is the inevitable result of the individual self-system development, and it was restricted by the structure 
and process of self-system. The influence factors to autonomous learning process includes planning, setting goals, selecting learning strategies, self-monitoring and selfevaluation, etc. The process of self-development level directly affects the quality of the independent learning process. Autonomous learning generally follows three steps: setting goals; making plan and choosing learning strategies; behavior execution and evaluation.

\section{The Information Processing View of Autonomous Learning}

The information processing theory of autonomous learning is put forward by some researchers represented by Canadian psychologist S.H.Winne. The theory was using information processing cybernetics to explain the autonomous learning. It was assumed that autonomous learning relies on a loop feedback loop. The information obtained by the individual should preset test according to the presupposed standard. If the match is not sufficient, it will need to change or converse the information, and then it is repeated till the information conforms to the inspection standards. If it is up to standard, it will exit in the form of information output.

\section{The Social Cognitive Theory of Autonomous Learning}

Social learning theory represented by Bandura explains autonomous learning from the interaction of the behavior, the environment and the individual internal factors. The theory is that autonomous learning is essentially based on learning expected behavior, the contrast and evaluation between the plans and reality behavior to regulate and control the process of learning. Autonomous learning includes three specific processes: self-observation, self-judgment and self-reaction. Self-observation refers to the students observe and understand their own learning behavior; self-judgment is giving the judgment and evaluation after comparing the learning results and learning standard; self-reaction is the inner experience or behavior based on the study of self-judgment and evaluation.

\section{E. The Autonomy Will View of Autonomous Learning}

The autonomy will view of autonomous learning is put forward by the German psychologist J.Ku2hl and American psychologist L.Corno. This theory thought that the students' autonomous learning process is actually a kind of will controlling process, which stressed the learner is the main body and the performer of action. Corno. Thought autonomous learning process could be divided into implicit self-control (including cognitive monitoring, mood monitoring and incentive monitoring) and explicit self-control (including things controlling and task controlling in learning environment).

\section{F. The Words Guidance Concept of Autonomous Learning}

The D column lu school represented by Vygotsky thought that autonomous learning is essentially a self-directed speech process, and it is an active process to adjust their learning using internal speech by the individual. They divided the children's speech development into three stages from low to high: external words, self-centered words and inner speech. And it pointed out that, in terms of children's learning activities, the external speech stage is mainly guided and controlled by the words of the outside society members; in the self-centered words stage they mainly adjust by their own words, that is the self-centered words; and in the stage of inner speech, learning is mainly guided and controlling by their internal silence words. So autonomous learning actually is the result of children's speech internalization.

\section{G. The Cognitive Constructivism Concept of Autonomous Learning}

The cognitive constructivism school represented by Flavel believes that autonomous learning is, in fact, metacognitive monitoring learning, and it is a process for students to actively adjust learning strategies and efforts according to their learning ability and learning task. Autonomous learning requires the individual to have conscious awareness and response to why to learn, whether to learn or not, what to learn and how to learn

\section{The STUdy of Autonomous LEARNING INFLUENCE FACTORS}

From the perspective of influence factors, it is generally believed that the factors affecting autonomous learning come from both individual internal and individual external. From the individual internal, learners' attitudes, beliefs and cognitive process are mentioned in several studies. At the same time, the learner's ability, self-efficacy, self-observation, self-judgment, self-reaction and the using of learning strategies directly affect the development of autonomous learning. In many factors from individual external, it is thought that the school's teaching management system plays an important role to autonomous learning. Besides the indirect influence to students' autonomous learning from formulating the talent training target, the talent training scheme, teaching and student management system, it also has direct impact on students' autonomous learning by requirements that put forward clearly based on the learning objective, attitudes and listening, self-study, evaluation and evaluation in the process of learning activities. In addition, the external influence factors include social culture, material, the relationship between teachers and students, and companion. About the factors that affect college students' autonomous learning, the researchers found that students with high selfconcept are more active in learning (Huang Shiwei, 2002). Other researchers have found that the self-concept not only has a direct influence on autonomous learning, but it also works on autonomous learning through learning attribution and learning environment adapting; And the learning environment adapting affects autonomous learning through learning attribution; And the harder students tend to do the internal attribution, the more beneficially the autonomous learning develops. (Yin Min, The observation and study analysis about college students' autonomous learning affecting factors, Psychology Journal, 2007). But on the whole, the research on the influence factors of college students' autonomous learning remains to be deepened.

\section{The ReAnalysis of College Students' AutONOMOUS LEARNING INFLUENCE FACTORS}

On the basis of previous studies, we put in this paper the cognitive constructivism theory of autonomous learning as the theoretical basis, using the grounded theory in qualitative 
research and interviewing a lot of college students in the form of semi-structured interview subjects. We hope to find a model assumes of autonomous learning affecting factors theory, so as to expand the research perspective of this field and provide a reference for the putting forward of effective education strategy.

The interview object of this study includes undergraduates in the universities administrated by the Ministry of Education as well as in the provincial universities. Interview content mainly includes: (1)to talk about the present study situation and how much pressure on study (including the difficulty of the course content and the homework) (2)how to arrange the independent time (3)do you have learning goals, have you thought about the future and what is the difference between having goals and no target? (4)what do you think of your autonomous learning ability and do you think what factors may affect the autonomous learning? (5)what to do if you don't want to learn? (6)what do you think of the relationship among autonomous learning, learning atmosphere and students? Why? (7)to talk about your classmates around. Besides the above questions in interview process for the interviewees, the independent problems can also be appropriate. Interviewer can also focus on one problem for trace asking.

By sorting all the interviews and merging similar answers, we come to a double-facet conclusion: the first one is in terms of the individual internal factors, the main content of the interviewee involves learning motivation which includes will, learning interest and self-efficacy; introspection which includes goal setting, feedback and positioning; self-regulation, and the most is the willpower. The second one is from the external environment, it is mainly related to environmental stress which includes academic pressure, employment pressure; peer influence (contrast) which includes other people's ideas to the future and the plan for the future; opportunity showing, from that the interviewees think this aspect can also affect the autonomous learning effectively, and they think the showing is the platform embodying the own ability. To achieve a good effect, they will be hard to prepare, so as to promote autonomous learning.

College students' autonomous learning is a complex process, and during the process, various factors are likely to impact on the autonomous learning. The influencing factors are from the external or internal, and they are active or passive. External cause works through internal. In addition to confirming the previous research conclusion, this study has also findings in the following respects. From the interview results, it is found that many participants stressed their own important positioning. Only clear personal locator (or self-evaluation), people can know what to want and have self-requirements. In the process, the willpower has played an important role in maintaining behavior and removing interference. The people with weak willpower embodies weaker in the self-learning. In the external influence factors, many participants are mentioned the contrast between partners. The contrast reflects on the ideal and world view personal holding, which is more obvious in the universities administrated by the Ministry of Education. When you see the classmates are in the struggle for the noble ideal, it virtually also increases the power of autonomous learning. In addition, whether there is a self-showing platform or not is also considered the important factor affecting autonomous learning.
If the learning process is the process of machining material, then the external performance will be to examine the effects of learning process. Many participants said, a display platform for them is the good motivation to promote autonomous learning. Although it seems to be related with the external motivation, it will play a twice the result with half the effort when we can combine the external motivation and internal motive. In this paper, the study gets the following autonomous learning theory model assumption:

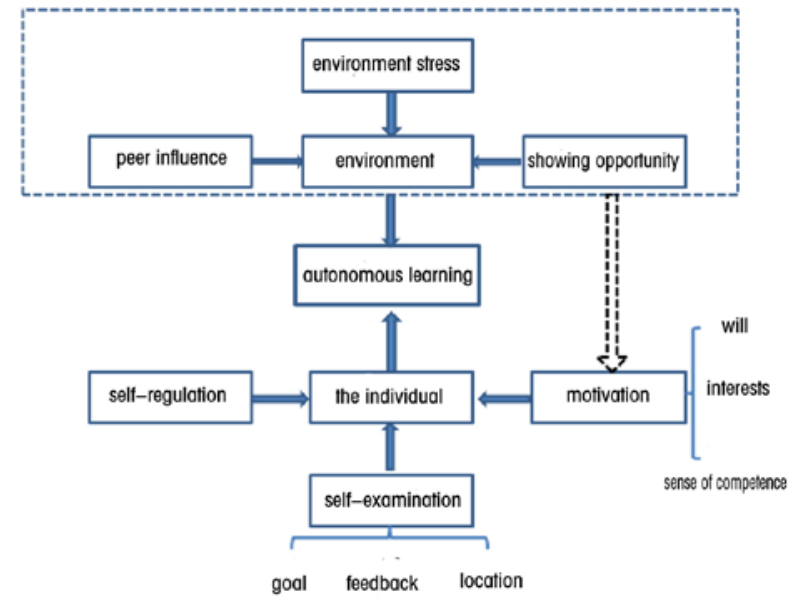

Fig. 1. Autonomous learning theory model

\section{The Strategy OF IMPROVIng COLlEge StUdentS' AUTONOMOUS LEARNING}

\section{A. The Exogenous Strategy}

As the manager, the school should provide students with a special place which is helpful to form good autonomous learning atmosphere. Especially it should create the platform allowing students to show their talent and make them realize the knowledge learning in school useful. Designing course should consider the students' independent time, leaving space for students' autonomous learning, and it should make the students have the certain free time, providing time for autonomous learning. Also it should attach great importance to the learning atmosphere construction, make full use of the model power, and with vivid examples and appropriate methods pay attention to the publicity and education to the ideal and faith. Teachers in teaching can be referred to Flipped Classroom teaching mode to supervise the students' autonomous learning, which makes the classroom a place for negotiating and discussing cooperation between students and students, students and teachers.

\section{B. The Endogenous Strategy}

As the main body of learning, students must firstly have a clear understanding to themselves. They should have the correct evaluation about their abilities, have the objective positioning, not blindly accept the learning, and learn their own professional advantage and the employment direction. What's more they should have a clear goal for their own future and hope, cultivating their interests in learning and dedicated spirit. Interest is the internal motivation to active learning. Only when 
interested in one thing, the individual is to further explore. So it needs to further analyze oneself, finding one’s interests.

In addition, it needs to strengthen the willpower exercise. The will is the psychological process in which the individual consciously overcomes the difficulties to achieve the expected goal. Lack of willpower will make it difficult to maintain longterm of autonomous learning. Therefore, to strengthen the willpower exercise is an effective way to ensure the effective in autonomous learning.

Making good self-regulation. First of all, have the reasonable arrangement of study time. Divide the learning objectives into short, medium and long-term goals and according to the actual situation make the semester plan and week plan and strictly enforcing them. A clear daily plan can guarantee the time of autonomous learning. In addition to the clear plan, it also needs introspection, and through introspection people can adjust the unreasonable plan. Second, adjust the negative emotions. Emotions directly affect the efficiency of individual activities, and positive emotion can improve the learning performance, while negative emotions can reduce the learning performance. Therefore, to ensure the effectiveness of autonomous learning, it needs to struggle against the negative emotions. To conquer negative emotions, people can use relaxing therapy, exercise, and talk, apply selfsuggestion and change the unreasonable cognition method.

\section{REFERENCES}

[1] Yan Ming, The Training Mode Research on College English Autonomous Learning Ability[M]. Harbin: Heilongjiang university press,2009:2

[2] Wang Jingqiong, Zhang Wei, The Mediation Effect Model of College Students' Autonomous Learning Affecting Factors[J]. Psychology Journal,2010,Vol.42

[3] Zhang Yufang, Lei Tinan, The Reasearch on College Students' Autonomous Learning based on MOOC[J], Journal of Hunan University College, 2015:1

[4] Wu Yuanyuan, The Research of University Undergraduate Extracurricular Autonomous Learning in China[D], Graduate thesis, 2011

[5] Zhang Jing, Tian Fengrong, The Research Progress of Autonomous Learning Affecting Factors[J], Social Psychology, 2012:Z1 